\title{
Cardiopulmonary recovery after COVID-19: an observational prospective multicentre trial
}

\author{
Thomas Sonnweber (101,16, Sabina Sahanic ${ }^{1,16}$, Alex Pizzini (1) ${ }^{1}$, Anna Luger², \\ Christoph Schwabl ${ }^{2}$, Bettina Sonnweber ${ }^{3}$, Katharina Kurz (1) ${ }^{1}$, \\ Sabine Koppelstätter ${ }^{1}$, David Haschka ${ }^{1}$, Verena Petzer ${ }^{4}$, Anna Boehm (1) ${ }^{1}$, \\ Magdalena Aichner ${ }^{1}$, Piotr Tymoszuk (1) ${ }^{1}$, Daniela Lener (i) $^{5}$, Markus Theurl ${ }^{5}$, \\ Almut Lorsbach-Köhler ${ }^{1}$, Amra Tancevski (10 ${ }^{1}$, Anna Schapfl ${ }^{3}$, Marc Schaber ${ }^{3}$, \\ Richard Hilbe ${ }^{1}$, Manfred Nairz ${ }^{1}$, Bernhard Puchner ${ }^{6}$, Doris Hüttenberger ${ }^{1}$, \\ Christoph Tschurtschenthaler ${ }^{1}$, Malte Aßhoff ${ }^{1}$, Andreas Peer ${ }^{7}$, Frank Hartig ${ }^{7}$, \\ Romuald Bellmann ${ }^{7}$, Michael Joannidis ${ }^{7}$, Can Gollmann-Tepeköylü $\mathbb{1}^{8}$, \\ Johannes Holfeld ${ }^{8}$, Gudrun Feuchtner $\mathbb{1}^{2}$, Alexander Egger ${ }^{9}$, Gregor Hoermann ${ }^{9,10,11}$, \\ Andrea Schroll ${ }^{1}$, Gernot Fritsche ${ }^{1}$, Sophie Wildner ${ }^{1}$, Rosa Bellmann-Weiler ${ }^{1}$, \\ Rudolf Kirchmair ${ }^{5,6}$, Raimund Helbok ${ }^{12}$, Helmut Prosch (10 ${ }^{13}$, Dietmar Rieder ${ }^{14}$, \\ Zlatko Trajanoski ${ }^{14}$, Florian Kronenberg ${ }^{15}$, Ewald Wöll ${ }^{3}$, Günter Weiss (1) ${ }^{1}$,

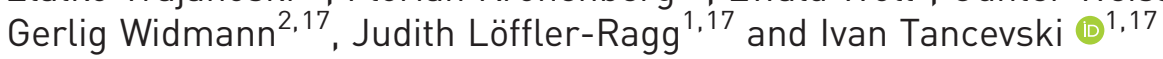

@ERSpublications

100 days after COVID-19 onset, a high portion of patients exhibit persisting symptoms and cardiopulmonary impairment. Still, a marked improvement of symptoms, pulmonary function and CT pathologies was found at follow-up. https://bit.ly/35YP13g

Cite this article as: Sonnweber T, Sahanic S, Pizzini A, et al. Cardiopulmonary recovery after COVID-19: an observational prospective multicentre trial. Eur Respir J 2021; 57: 2003481 [https://doi.org/10.1183/ 13993003.03481-2020].

\section{ABSTRACT}

Background: After the 2002/2003 severe acute respiratory syndrome outbreak, 30\% of survivors exhibited persisting structural pulmonary abnormalities. The long-term pulmonary sequelae of coronavirus disease 2019 (COVID-19) are yet unknown, and comprehensive clinical follow-up data are lacking.

Methods: In this prospective, multicentre, observational study, we systematically evaluated the cardiopulmonary damage in subjects recovering from COVID-19 at 60 and 100 days after confirmed diagnosis. We conducted a detailed questionnaire, clinical examination, laboratory testing, lung function analysis, echocardiography and thoracic low-dose computed tomography (CT).

Results: Data from 145 COVID-19 patients were evaluated, and $41 \%$ of all subjects exhibited persistent symptoms 100 days after COVID-19 onset, with dyspnoea being most frequent (36\%). Accordingly, patients still displayed an impaired lung function, with a reduced diffusing capacity in $21 \%$ of the cohort being the most prominent finding. Cardiac impairment, including a reduced left ventricular function or signs of pulmonary hypertension, was only present in a minority of subjects. CT scans unveiled persisting lung pathologies in $63 \%$ of patients, mainly consisting of bilateral ground-glass opacities and/or reticulation in the lower lung lobes, without radiological signs of pulmonary fibrosis. Sequential follow-up evaluations at 60 and 100 days after COVID-19 onset demonstrated a vast improvement of symptoms and CT abnormalities over time.

Conclusion: A relevant percentage of post-COVID-19 patients presented with persisting symptoms and lung function impairment along with radiological pulmonary abnormalities $>100$ days after the diagnosis of COVID-19. However, our results indicate a significant improvement in symptoms and cardiopulmonary status over time.

Copyright OERS 2021. This version is distributed under the terms of the Creative Commons Attribution NonCommercial Licence 4.0. 


\section{Introduction}

Coronavirus disease 2019 (COVID-19) shares clinical and mechanistic characteristics with the severe acute respiratory syndrome (SARS) outbreak of 2002/2003, including angiotensin-converting enzyme-2-dependent cellular entry and interleukin (IL)-6-driven hyperinflammation, potentially leading to imbalanced immune responses and acute respiratory distress syndrome (ARDS) [1-13]. In different studies evaluating SARS survivors months after infection, fibrotic features, including abnormal scoring of airspace opacity and reticular shadowing, were observed in up to $36 \%$ of the patients $[2,14,15]$. A 1-year follow-up study on 97 recovering SARS patients in Hong Kong showed that $27.8 \%$ of survivors presented with decreased lung function and signs of pulmonary fibrosis compared to a normal population, which was confirmed by another follow-up study $[2,14,16]$. Moreover, the phylogenetically related Middle East respiratory syndrome (MERS) coronavirus $(\mathrm{CoV})$ was shown to induce pulmonary fibrosis in up to $33 \%$ of patients [17]. As SARS-CoV-2 shares high homology with SARS-CoV-1, and to a lesser extent with MERS-CoV, it is thus conceivable that survivors of COVID-19 may also develop pulmonary fibrosis [7]. With worldwide $>74$ million confirmed cases at the time of writing, and an average $20 \%$ of patients with a moderate-to-severe course of infection often needing hospitalisation, the development of fibrosing lung disease after clearance of infection could become an enormous health concern [1].

We thus designed a prospective, multicentre, observational study, the Development of Interstitial Lung Disease (ILD) in Patients with SARS-CoV-2 infection (CovILD) study, aimed at systematically evaluating the persisting cardiopulmonary damage of COVID-19 patients 60 days and 100 days after COVID-19 onset.

\section{Methods}

\section{Study design}

Enrolment of the CovILD study began on April 29, 2020 (supplementary figure S1). The trial site is located at the Department of Internal Medicine II, Medical University of Innsbruck (Innsbruck, Austria), with two additional participating medical centres in Zams and Münster (Austria), which are tertiary care centres (Innsbruck, Zams) and an acute rehabilitation facility (Münster) all located in Tyrol, the first major COVID-19 hotspot in Austria. Diagnosis of COVID-19 was confirmed if a typical clinical presentation (according to current World Health Organization guidelines) along with a positive reverse transcriptase PCR SARS-CoV-2 test obtained from a nasopharyngeal or oropharyngeal swab were present [18]. Eligibility included the necessity of hospitalisation (either on a normal ward or intensive care unit (ICU)) or outpatient care with persisting symptoms. A total of 190 patients were screened for study participation and 145 individuals were included in the final study. Reasons for nonparticipation were mainly logistic (e.g. tourists who left the country, and individuals who lived too far away from the study centre in Innsbruck to attend regular follow-up; $\mathrm{n}=27)$ or rejection of study participation $(\mathrm{n}=18)$.

We present a follow-up evaluation performed 60 days (mean \pm SD $63 \pm 23$ days; visit 1 ) and 100 days (103 \pm 21 days; visit 2) after diagnosis of COVID-19. Of note, in Tyrol the healthcare system was never overloaded at the local peak of the pandemic, thus, all patients received supportive care according to the standard of care at the trial site hospitals and no selection bias due to triage methods was apparent. The trial protocol was

This article has an editorial commentary: https://doi.org/10.1183/13993003.04423-2020

This article has supplementary material available from erj.ersjournals.com

Received: 11 Sept 2020 | Accepted: 18 Nov 2020

Affiliations: ${ }^{1}$ Dept of Internal Medicine II, Medical University of Innsbruck, Innsbruck, Austria. ${ }^{2}$ Dept of Radiology, Medical University of Innsbruck, Innsbruck, Austria. ${ }^{3}$ Dept of Internal Medicine, St Vinzenz Hospital, Zams, Austria. ${ }^{4}$ Dept of Internal Medicine V, Medical University of Innsbruck, Innsbruck, Austria. ${ }^{5}$ Dept of Internal Medicine III, Medical University of Innsbruck, Innsbruck, Austria. ${ }^{6}$ The Karl Landsteiner Institute, Reha Zentrum Münster, Münster, Austria. ${ }^{7}$ Division of Intensive Care and Emergency Medicine, Dept of Internal Medicine I, Medical University of Innsbruck, Innsbruck, Austria. ${ }^{8}$ Dept of Cardiac Surgery, Medical University of Innsbruck, Innsbruck, Austria. ${ }^{9}$ Central Institute of Medical and Chemical Laboratory Diagnostics, University Hospital Innsbruck, Innsbruck, Austria. ${ }^{10}$ Dept of Laboratory Medicine, Medical University of Vienna, Vienna, Austria. ${ }^{11}$ MLL Munich Leukemia Laboratory, Munich, Germany. ${ }^{12}$ Dept of Neurology, Medical University of Innsbruck, Innsbruck, Austria. ${ }^{13}$ Dept of Biomedical Imaging and Imageguided Therapy, Medical University Vienna, Vienna, Austria. ${ }^{14}$ Institute for Bioinformatics, Medical University of Innsbruck, Innsbruck, Austria. ${ }^{15}$ Institute of Genetic Epidemiology, Medical University of Innsbruck, Innsbruck, Austria. ${ }^{16}$ Contributed equally as first authors. ${ }^{17}$ Contributed equally to this article as lead authors and supervised the work.

Correspondence: Judith Löffler-Ragg, Anichstraße 35, 6020 Innsbruck, Austria.

E-mail: judith.loefflerai-med.ac.at 
approved by the institutional review board at Innsbruck Medical University (EK Nr: 1103/2020) and was registered at ClinicalTrials.gov (registration number NCT04416100). Informed consent was obtained from each patient.

\section{Procedures}

During the follow-up visits, the following examinations were performed: clinical examination; medical history; a questionnaire about typical COVID-19 symptoms including cardiorespiratory, gastrointestinal and neurological symptoms during the disease and at follow-up, the modified British Medical Research Council (mMRC) dyspnoea score, a standardised performance status score, lung function testing including spirometry, body plethysmography and diffusing capacity of the lung for carbon monoxide $\left(D_{\mathrm{LCO}}\right)$ adjusted for haemoglobin, capillary blood gas analysis, transthoracic echocardiography, standard laboratory examinations and a low-dose computed tomography (CT) scan of the chest.

All serological markers were determined on fully automated random access instruments: C-reactive protein (CRP), IL-6, procalcitonin (PCT), N-terminal pro-brain natriuretic peptide (NT-proBNP) and serum ferritin were determined on a Roche Cobas 8000 analyser (Basel, Switzerland) and D-dimer on a Siemens BCS-XP instrument using the Siemens D-Dimer Innovance reagent (Erlangen, Germany).

CT scans were acquired without ECG gating on a 1280slice multidetector CT with a $128 \times 0.6 \mathrm{~mm}$ collimation and spiral pitch factor of 1.1 (SOMATOM Definition Flash, Siemens Healthineers). Scans were obtained in the craniocaudal direction without iodine contrast agent and in a low-dose setting $(100 \mathrm{kVp}$ tube potential). If patients had a clinical suspicion for pulmonary embolism (PE), additional contrast CTs were conducted. Axial reconstructions were performed with a slice thickness of $1 \mathrm{~mm}$. CT images were evaluated for the presence of ground-glass opacities (GGOs), consolidations, bronchiectasis and reticulations as defined by the glossary of terms of the Fleischner Society [19]. When present, the distribution of the findings was graded according to their distribution (unilateral/bilateral, involved lobes). Overall, pulmonary findings were graded for every lobe using the following CT severity score: 0: none; 1: minimal (subtle GGOs); 2: mild (several GGOs, subtle reticulation); 3: moderate (multiple GGOs, reticulation, small consolidation); 4: severe (extensive GGOs, consolidation, reticulation with distortion); and 5: massive (massive findings, parenchymal destructions). The maximum score was 25 (i.e. maximum score 5 per lobe). This score was used because the British Society of Thoracic Imaging (BSTI) definition of COVID-19 classifies only the extent of abnormality $(<25 \%, 26-50 \%, 51-75 \%,>75 \%)$, and the BSTI post-COVID-19 CT report codes do not discriminate between "improving" (PCVCT1: no significant fibrosis or concerning features) and "fibrosis" (РCVCT3: fibrosis \pm inflammatory change present (inflammation $>$ fibrosis/fibrosis $>$ inflammation/fibrosis without inflammation)). In addition, we did not have a pre-COVID-19 baseline and an acute phase COVID-19 CT scan in most patients.

The Syngo.via CT Pneumonia Analysis (Siemens Healthineers) research prototype for the detection and quantification of abnormalities consistent with pneumonia was used to calculate the percentage of opacity and percentage of high opacity, indicating percentages of GGOs and consolidation, respectively.

\section{Statistical analysis}

Statistical analyses were performed using statistical analysis software package (IBM SPSS Statistics version 24.0; IBM, Armonk, NY, USA). According to descriptive statistical analysis including tests for homoscedasticity and data distribution (Levene test, Kolmogorov-Smirnov test, Shapiro-Wilk test and density blot/histogram analysis) two-sided parametric or nonparametric tests were applied as appropriate. For group comparisons of continuous data, the Mann-Whitney U-test, Kruskal-Wallis or Wilcoxon signed-rank test were applied. Binary and categorical data were analysed with Fisher's exact test, Chi-squared test or McNemar test. Multiple testing was adjusted by the Sidak formula. Correlations were assessed with Spearman rank (nonparametric data) or Pearson's (parametric data) test. To identify demographic and clinical factors impacting on the persistence of symptoms and radiological lung findings at follow-up, a series of fixed-effect ordinary and generalised linear models were created using R programming suite version 3.6.3 (www.r-project. org). Details of the statistical analysis and the software packages used are reported in the supplementary methods. Independently of the testing technique, effects were termed significant for $\mathrm{p}<0.05$.

\section{Results}

\section{Characteristics of the cohort}

In total, 145 patients with a confirmed diagnosis of COVID-19 participated in the CovILD study, and 133 subjects were also available for the second follow-up. The mean \pm SD time from the diagnosis of COVID-19, as defined by positive SARS-CoV-2 PCR testing, to follow-up was $63 \pm 23$ days for visit 1 and $103 \pm 21$ days for visit 2. The study cohort consisted of 55\% male individuals, aged 19-87 years (table 1). 61\% of COVID-19 patients were overweight or obese (body mass index $>25 \mathrm{~kg} \cdot \mathrm{m}^{-2}$ or $>30 \mathrm{~kg} \cdot \mathrm{m}^{-2}$, respectively). 


$\begin{array}{lc}\text { TABLE } 1 \text { Demographics and clinical characteristics of patients enrolled in the Development of } \\ \text { Interstitial Lung Disease (ILD) in Patients with SARS-CoV-2 infection (CovlLD) study } \\ \text { Subjects } & 145 \\ \text { Age years } & 57 \pm 14 \\ \text { Female } & 63(43) \\ \text { BMI kg-m }{ }^{-2} & 26 \pm 5 \\ \text { Smoking history } & 57(39) \\ \text { Current smokers } & 4(3) \\ \text { Pack-years } & 8 \pm 16 \\ \text { Comorbidities } & \\ \text { None } & 33(23) \\ \text { Cardiovascular disease } & 58(40) \\ \text { Hypertension } & 44(30) \\ \text { Pulmonary disease } & 27(19) \\ \quad \text { COPD } & 8(6) \\ \quad \text { Asthma } & 10(7) \\ \text { ILD" } & 1(1) \\ \text { Metabolic disease } & 63(43) \\ \quad \text { Hypercholesterolaemia } & 27(19) \\ \text { Diabetes mellitus, type } 2 & 24(17) \\ \text { Chronic kidney disease } & 10(7) \\ \text { Chronic liver disease } & 8(6) \\ \text { Malignancy } & 17(12) \\ \text { Immunodeficiency" } & 9(6) \\ \text { Hospitalised } & 109(75) \\ \text { In-hospital treatment } & + \\ \text { Oxygen supply } & 72(66) \\ \text { Noninvasive ventilation } & 3(3) \\ \text { Invasive ventilation } & 29(27)\end{array}$

Data are presented as $n$, mean \pm SD or $n(\%)$. BMI: body mass index. \#: $n=1$ with a history of radiation-induced pneumonitis; ": due to disease or ongoing immunosuppressive treatment: renal transplantation $(n=1)$, psoriasis vulgaris $(n=1)$, morbus Hashimoto $(n=1)$, leukaemia $(n=1)$, lymphoma $(n=1)$, gout $(n=1)$, polyarthritis $(n=1) .{ }^{+}$: all patients needing noninvasive or invasive ventilation were supplied with oxygen before intensive care unit admission; relative numbers depict the treatment of in-hospital patients.

Most individuals had pre-existing comorbidities (77\%) with cardiovascular and metabolic diseases being the most frequent (table 1). The majority of study participants (75\%) were hospitalised during the acute phase of COVID-19, half of the hospitalised patients required oxygen supply, and $22 \%$ of all subjects were admitted to the ICU due to the necessity of noninvasive or invasive mechanical ventilation as determined by the treating physicians. Patients who were admitted to the ICU had more comorbidities such as cardiovascular disease, arterial hypertension, diabetes mellitus, hypercholesterolaemia, chronic kidney disease or immune deficiency as compared to subjects without the necessity of ICU treatment (supplementary table S1). Additionally, higher age and male sex were related to a more severe course of acute COVID-19.

\section{Clinical evaluation at follow-up}

At the second follow-up visit, a relevant number of patients still reported an impaired performance status and persisting symptoms including dyspnoea (36\%), night sweats (24\%), sleep disorders (22\%) or hyposmia/anosmia (19\%), but with decreasing frequency compared to the acute phase of COVID-19 and the first follow-up visit (figure 1). Notably, severe symptoms, such as a severely impaired performance status (as assessed by a standardised questionnaire) or severe dyspnoea (mMRC score 3-4) were only found in $2 \%$ and $4 \%$, respectively, of all study participants at second follow-up. Overall, a marked and continuous improvement of all assessed symptoms (namely impairment of performance status, dyspnoea, cough, fever, diarrhoea or vomiting, night sweats, hyposmia/anosmia and sleep disorders) from disease onset to follow-up at visits 1 and 2 was observed (figure 1).

\section{Cardiopulmonary evaluation at follow-up}

At follow-up visit 1 and visit 2, trans-thoracic echocardiography unveiled a high rate of diastolic dysfunction (60\% and 55\% of all subjects, respectively). Signs of pulmonary hypertension as well as a pericardial effusion were detected only in a smaller portion of the cohort (supplementary table S2). Only 
a)

Impairment of performance status

Persisting symptoms

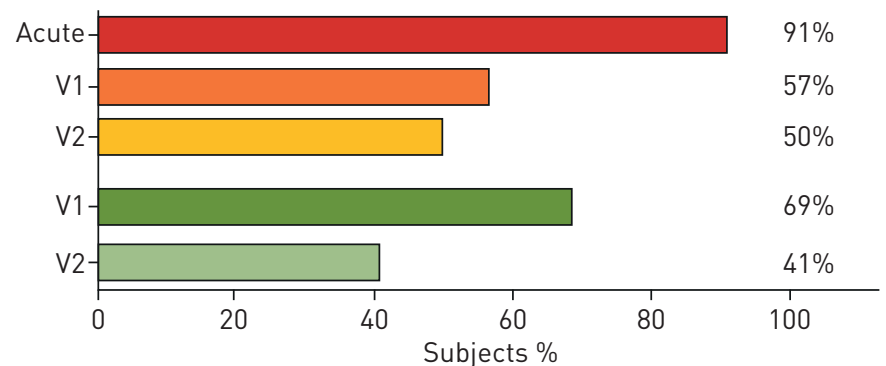

Acute COVID-19 Second follow-up after COVID-19

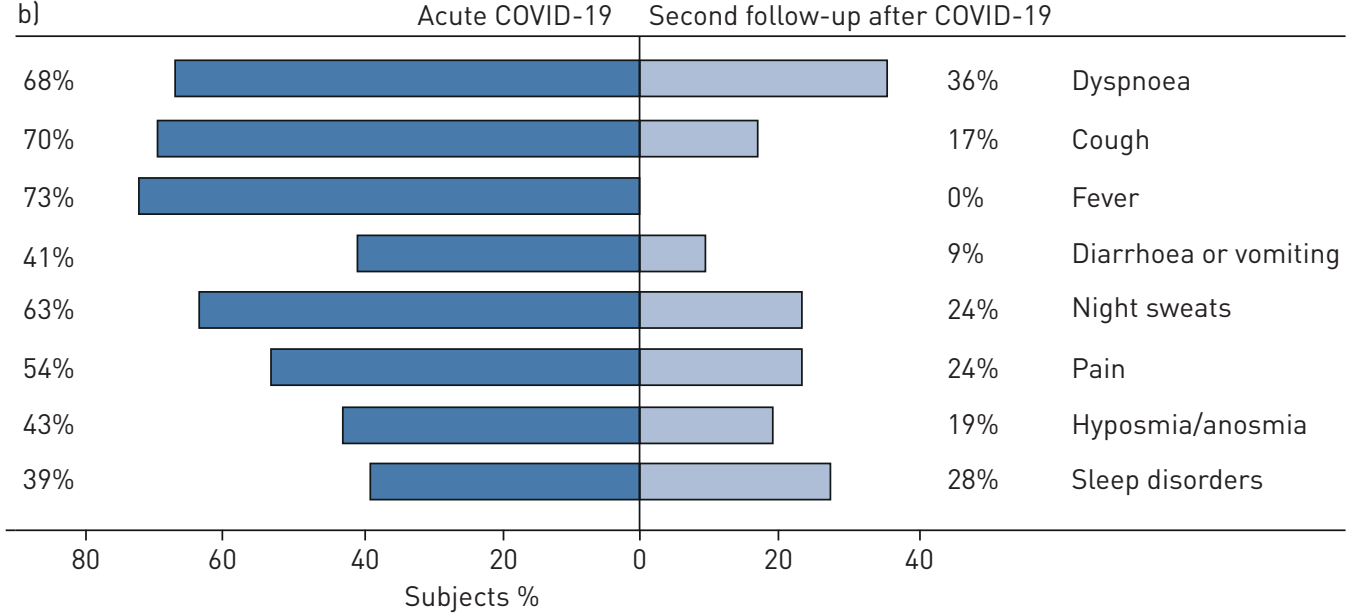

FIGURE 1 Symptom burden in the Development of Interstitial Lung Disease (ILD) in Patients with SARS-CoV-2 infection (CovlLD) study cohort during acute coronavirus disease 2019 (COVID-19) and at follow-up. a) Using a standardised questionnaire, performance status and overall burden of symptoms were assessed for the time-point of disease onset, 60 days (V1), and 100 days (V2) after diagnosis of COVID-19. b) Symptom burden was assessed using a standardised questionnaire at COVID-19 onset and at 100 days post-COVID-19 diagnosis. All symptoms significantly improved over time $\left(p<0.001\right.$ for all read-outs). $n_{\text {acute }}=145, n_{\text {follow-up }}=135$.

four participants presented with a reduced left ventricular ejection fraction (LVEF). Whereas the frequency of diastolic dysfunction, signs of pulmonary hypertension and LVEF impairment did not significantly change from follow-up visit 1 to visit 2 , the number of patients with pericardial effusion diminished over time $(\mathrm{p}=0.039)$.

An impaired lung function, reflected by reduced static and/or dynamic lung volumes, or impaired $D_{\text {LCO }}$, was present in $42 \%$ and $36 \%$ of individuals at visit 1 and visit 2, respectively (table 2). In detail, 100 days after diagnosis of COVID-19, a reduction in forced vital capacity (FVC) and/or forced expiratory volume in $1 \mathrm{~s}\left(\mathrm{FEV}_{1}\right)$ was found in $22 \%$, a reduced TLC in $11 \%$ and an impaired $D_{\mathrm{LCO}}$ in $21 \%$ of all individuals. Additionally, hypoxia, as reflected by a reduced oxygen tension $\left(P_{\mathrm{O}_{2}}\right)$ assessed with capillary blood gas analysis $\left(P_{\mathrm{O}_{2}}<75 \mathrm{mmHg}\right.$ ), was still present in $37 \%$ of all subjects, and these patients demonstrated mild (80\%, $\left.P_{\mathrm{O}_{2}}<75-65 \mathrm{mmHg}\right)$ or moderate $\left(20 \%, P_{\mathrm{O}_{2}}<65-55 \mathrm{mmHg}\right)$ hypoxia at rest. Notably, dynamic lung volumes and $D_{\text {LCO }}$ significantly increased over time. As compared to follow-up visit 1 there was a moderate but significant improvement of most of these parameters over time (table 2).

\section{Serological markers}

At visit 2, mild elevations in inflammatory markers such as CRP (12\%), IL-6 (6\%) or PCT (9\%) were still present in a smaller portion of the cohort (supplementary table S3). Accordingly, biomarkers associated with COVID-19 disease severity, such as D-dimer, NT-proBNP and serum ferritin were still elevated in $27 \%, 23 \%$ and $17 \%$ of COVID-19 patients, respectively, at second follow-up.

\section{Pulmonary imaging}

CT imaging revealed radiological lung abnormalities typical for COVID-19 in 77\% of patients at visit 1 and in $63 \%$ of individuals at visit 2 (figure $2 \mathrm{a}$ ). The main findings were GGOs, consolidation and reticulation. Bronchial dilation was found in a small portion of the cohort (representative CT scans are depicted in figure 3). In $75 \%$ of patients, pulmonary involvement was found bilaterally, with the lower lobes most prominently affected (supplementary table S4). Notably, by the time of visit 2, consolidations and bronchial dilations almost completely resolved, and the mean extent of GGOs significantly decreased. 
TABLE 2 Pulmonary function of coronavirus disease 2019 (COVID-19) patients at follow-up

\begin{tabular}{|c|c|c|c|}
\hline & First follow-up" & Second follow-up" & $\mathrm{p}$-value time change \\
\hline Subjects & 126 & 133 & \\
\hline Lung function impaired ${ }^{+}$ & $53(42)$ & $48(36)$ & 0.388 \\
\hline FVC L & $3.6 \pm 1.0$ & $3.7 \pm 0.9$ & $<0.001$ \\
\hline FVC $<80 \%$ predicted & 34 (27) & $29(22)$ & 0.049 \\
\hline $\mathrm{FEV}_{1} \mathrm{~L}$ & $2.9 \pm 0.8$ & $3.0 \pm 0.8$ & 0.001 \\
\hline FEV $_{1}<80 \%$ predicted & $28(22)$ & $30(22)$ & 1.000 \\
\hline FEV $1 /$ FVC $\%$ & $84 \pm 11$ & $80 \pm 11$ & $<0.001$ \\
\hline $\mathrm{FEV}_{1} / \mathrm{FVC}<70 \%$ & $5(4)$ & $11(8)$ & 0.063 \\
\hline TLC L & $6.2 \pm 1.3$ & $6.2 \pm 1.3$ & 0.881 \\
\hline TLC $<80 \%$ predicted & $14(11)$ & $15(11)$ & 0.791 \\
\hline$D_{\text {Lco }} \mathrm{mmol} \cdot \mathrm{min}^{-1} \cdot \mathrm{kPa}^{-1}$ & $7.7 \pm 2.4$ & $7.9 \pm 2.3$ & $<0.001$ \\
\hline$D_{\text {Lco }}<80 \%$ predicted & $39(31)$ & $28(21)$ & 0.022 \\
\hline$P_{\mathrm{O}_{2}} \mathrm{mmHg}$ & $79 \pm 10$ & $78 \pm 9$ & 0.864 \\
\hline$P_{\mathrm{O}_{2}}<75 \mathrm{mmHg}$ & $40(32)$ & 45 (37) & 0.871 \\
\hline
\end{tabular}

Data are presented as $\mathrm{n}, \mathrm{n}(\%)$ or mean \pm SD, unless otherwise stated. Bold type represents statistical significance. Wilcoxon signed-rank test and McNemar test were used to assess time-related differences. FVC: forced vital capacity; $\mathrm{FEV}_{1}$ : forced expiratory volume in $1 \mathrm{~s}$; TLC: total lung capacity; $D_{\text {LCo }}$ : diffusing capacity of the lung for carbon monoxide; $P_{\mathrm{O}_{2}}$ : partial pressure of oxygen assessed with blood gas analysis (without oxygen supplementation). \#: 60 days after COVID-19 diagnosis; ๆ: 100 days after COVID-19 diagnosis; ${ }^{+}$: lung function was considered impaired if $\mathrm{FVC}, \mathrm{FEV}_{1}, \mathrm{FEV}_{1} / \mathrm{FVC}$ ratio, TLC or $D_{\text {LCo }}$ were below the predicted normal.

In contrast, reticulations only gradually improved from visit 1 to visit 2 (figure $2 \mathrm{a}$ ). In eight patients, who presented with a clinical suspicion of an incidental PE (e.g. deteriorating dyspnoea despite resolution of radiological lung findings, tachycardia, significant hypoxia or lack of D-dimer decrease/D-dimer increase), additional contrast agent CT was performed, and one incidental PE was detected.

Radiological assessment of the severity of COVID-19-related lung pathologies was performed on CT scans using both an automated software-based analysis with Syngo.via CT Pneumonia Analysis Software as well as an evaluation by three independent radiologists. Importantly, the software-based pneumonia quantification and the severity scoring by radiologists demonstrated a high correlation (correlation coefficient $\rho=0.893, p<0.001)$. The pulmonary CT severity score unveiled a moderate structural involvement in most patients at the first follow-up, which significantly improved over time (figures $2 \mathrm{~b}, \mathrm{c}$ and 4). The majority of participants (81\%) demonstrated an improvement in the CT severity score. Interestingly, we found only weak-to-moderate correlations of FVC $(\rho=0.322, p<0.001)$ and $\operatorname{FEV}_{1}(\rho=0.237$, $\mathrm{p}<0.001)$ measurements and moderate correlations of TLC $(\rho=0.455, \mathrm{p}<0.001)$ and $D_{\text {LCO }}(\rho=0.543, p<0.001)$ assessment with the CT severity score. The CT severity score $(\rho=0.200, p<0.001)$ and lung function parameters including FVC $(\rho=0.206, p<0.001)$, FEV $_{1}(\rho=0.212, p<0.001)$, TLC $(\rho=0.145, p<0.05)$ and $D_{\text {LCO }}$ $(\rho=0.204, p<0.001)$ demonstrated a weak correlation to the severity of dyspnoea.

Finally, we analysed the impact of demographic and clinical factors on the persistence of symptoms, patient performance status and CT findings at follow-up with a series of fixed-effect ordinary and generalised linear models (supplementary figures S2, S3 and S4). These analyses revealed that the severity of acute COVID-19 (as reflected by the need for medical treatment), age, sex and pre-existing diseases such as cardiovascular diseases, pulmonary diseases, diabetes mellitus type 2 and malignancy were related to patient recovery. Despite that, a striking improvement of the CT severity score was found in patients of all COVID-19 severity groups, including subjects who needed mechanical ventilation at an ICU (figure 5).

\section{Discussion}

The ongoing pandemic considerably increases the demand for healthcare and raises concerns on physiological and radiological disease outcome in COVID-19 patients needing hospitalisation or even treatment in the ICU [20]. Accordingly, early lung function analysis of patients with COVID-19 at the time of discharge from hospital revealed a high rate of abnormalities indicative of potential interstitial lung disease. Moreover, a retrospective study 30 days after discharge had shown a reduced diffusing capacity as the central finding in $26 \%$ of post-acute COVID-19 patients [21, 22]. To our knowledge, this prospective study represents the first 3-month cardiopulmonary follow-up analysis of patients with confirmed COVID-19 including critical cases. The majority of patients enrolled in the CovILD study were previously hospitalised, male and displayed comorbidities, which have been identified as risk factors for a severe 
a)
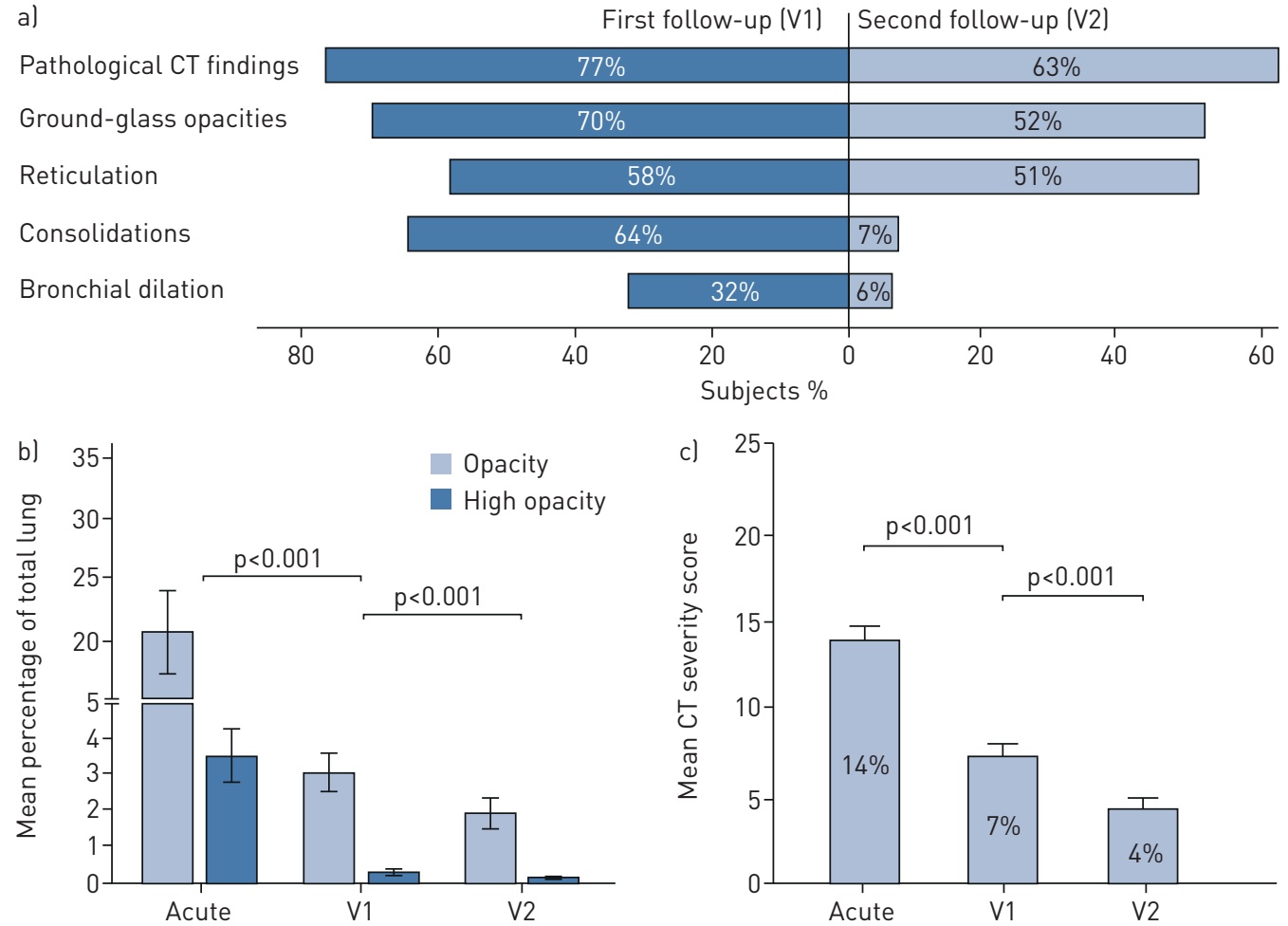

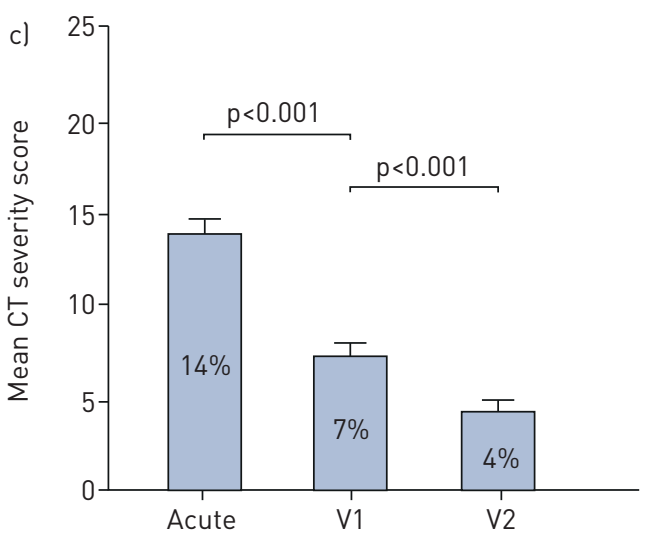

FIGURE 2 Chest computed tomography (CT) lung analysis at coronavirus disease 2019 (COVID-19) onset and follow-up. a) The pattern of pathological findings assessed with CT at 60 (V1) and 100 days (V2) after diagnosis of COVID-19. b) Automated analysis of lung opacities assessed on CT scans from the acute disease phase, 60 days and 100 days after COVID-19 diagnosis employing Syngo.via CT Pneumonia Analysis software (Siemens Healthineers, Erlangen, Germany). c) CT severity scoring by radiologists at COVID-19 onset, 60 days and 100 days after COVID-19 diagnosis. The severity score was calculated via CT evaluation by three independent radiologists who qualitatively graded lung impairment for each lobe separately (grade $0-5$, with 0 for no involvement and 5 for massive involvement). A total score was achieved by summation of grades for all five lobes (maximum 25 points). Data are presented as mean \pm SE. $n_{\text {acute }}=23, n_{v_{1}}=145, n_{v_{2}}=135$.

course of COVID-19 [23-26]. Importantly, $21 \%$ of patients were admitted to the ICU, comparable to the ICU admission rates of 26-33\% previously published for COVID-19 patients [1, 25].

During post-acute care, patients reported a high rate of persisting dyspnoea, and one-third of COVID-19 patients displayed an impaired lung function, with a reduced diffusing capacity being the most prominent finding even $>100$ days after COVID-19 diagnosis. This is in accordance with observations in 110 SARS survivors 3 and 6 months after infection [14]. Long-term SARS studies revealed disturbed gas exchange as the most relevant residual finding, ranging from $15.5 \%$ to $57.2 \%$ of patients $[2,14,27]$. Furthermore, several studies on ARDS survivors have shown that their pulmonary function generally returns to normal or near to normal within 6-12 months, and predominantly $D_{\text {LCO }}$ may remain abnormal in patients 1 year after recovery [28, 29]. Although a relevant portion of our cohort presented with persisting cardiopulmonary impairment at follow-up, as evidenced by echocardiography or lung function testing, we observed a gradual improvement over time.

The major CT findings of patients affected by COVID-19 were previously shown to include GGO and consolidation with bilateral involvement and peripheral and diffuse distribution [30]. Importantly, this study reveals that $>100$ days after disease onset, two-thirds of patients had residual radiological changes, predominantly including residual GGO combined with reticulation. Since most patients had no CT scan before or during hospitalisation, pre-existing lung injury cannot be completely ruled out for the subgroup of patients with moderate-to-severe findings present at follow-up.

In line with our findings, CT abnormalities with low mean severity were detected in $75.4 \%$ of SARS survivors 6 months after admission to the hospital [15]. From a clinical perspective, it is important to note that the high prevalence of persisting radiological abnormalities in our cohort was not well reflected by pulmonary function testing. Data on the inferiority of pulmonary function testing in the screening for ILD in a population at high risk for progressive disease support these findings [31]. To date, it remains unclear whether such subclinical structural changes justify the routine repetitive use of CT imaging in follow-up 

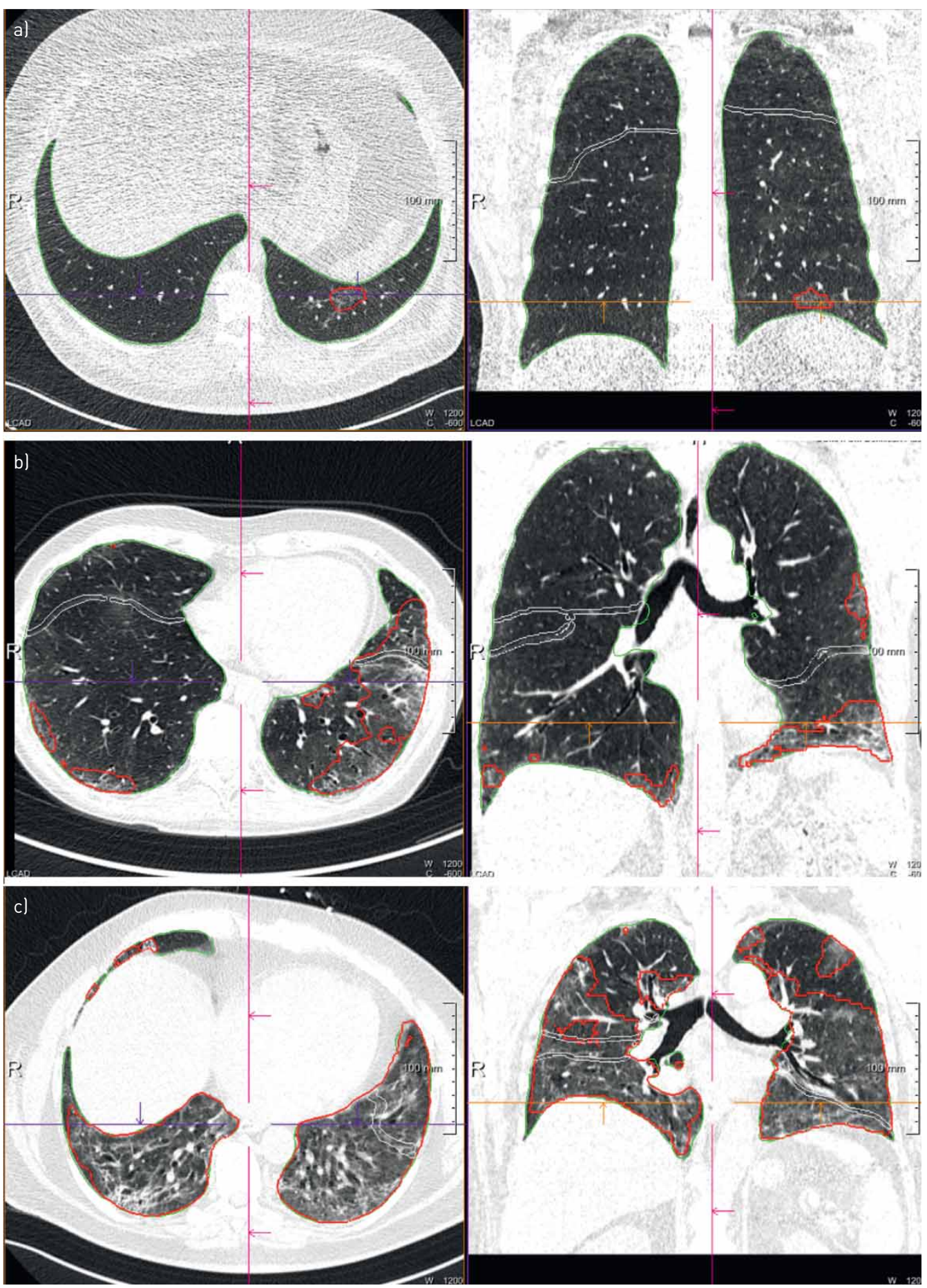

FIGURE 3 Representative computed tomography scans of coronavirus disease 2019 patients with a) minimal, b) moderate and c) severe radiological findings at first follow-up. Percentage of opacity/high opacity a) $0.07 /$ 0.00 ; b) $10.29 / 0.69$; c) $56.87 / 5.92$.

care after COVID-19 pneumonia. For example, follow up-studies in ARDS survivors including chest CT have shown that persistent radiographic abnormalities were of little clinical relevance [32]. In addition, a 15-years follow-up study of 71 patients with moderate-to-severe SARS showed that the rate of interstitial abnormalities declined remarkably within the first 2 years of recovery to only $4.6 \%$ residual lung injury [27]. In line with this, our imaging findings demonstrate high reversibility of COVID-19-associated cardiopulmonary damage, and no clear signs of progressive ILD have been identified so far. 

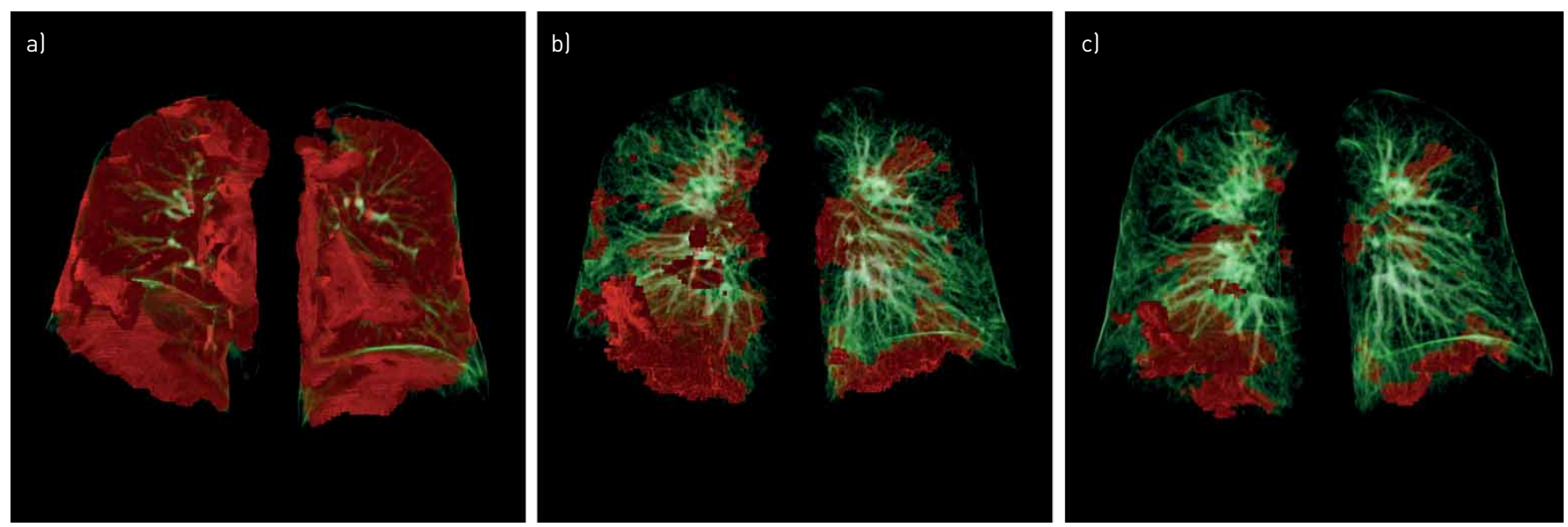

FIGURE 4 Representative sequential computed tomography (CT) scans of a 56-year-old male coronavirus disease 2019 (COVID-19) patient during acute disease and follow-up. Pulmonary three-dimensional modelling assessed with CT is shown al during acute COVID-19, b) at 60 days follow-up and c) at 100 days follow-up. Pulmonary opacities, mainly reflecting ground-glass opacities and/or consolidation, were quantified with Syngo.via CT Pneumonia Analysis software (Siemens Healthineers, Erlangen, Germany). Areas with increased opacity are marked in red, whereas normal lung areas are indicated in green.

Finally, we have to acknowledge limitations of the study presented. First, the CovILD trial was initiated during the onset of the COVID-19 epidemic in Austria; thus, pre-existing comorbidities were assessed retrospectively, and cardiopulmonary evaluation prior to COVID-19 was only available in a small portion of the cohort. Thus, we cannot fully evaluate the impact of potentially pre-existing cardiopulmonary impairment on the findings presented herein. Secondly, according to the ethics approval we used low-dose CT without contrast agent to assess radiological pulmonary impairment. Whereas this strategy reduces the cumulative radiation dose and precludes contrast agent related complications, it is also associated with limitations. Although the image quality of low-dose CT was found to be sufficient, some very subtle fibrotic changes may be recognised as (nonfibrotic) reticulations due to the lower image quality. However, even in regular-dose CT, clear discrimination between residual inflammation and early fibrosis is extremely difficult, especially in patients with a clear overall improvement. Additionally, as we did not routinely use contrast agent, we did not screen the cohort for occult PE, and additional contrast CTs were only indicated in case of clinical suspicion of PE.

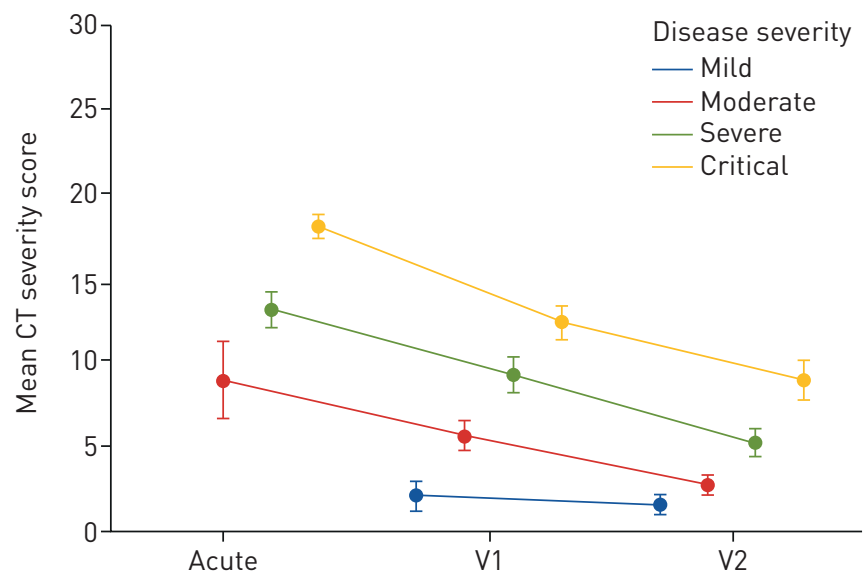

FIGURE 5 Changes in pulmonary impairment according to computed tomography (CT) analysis in patients of different acute coronavirus disease 2019 (COVID-19) disease severities. Time-dependent changes of CT severity score in patients with mild to critical COVID-19. Disease severity was graded by the need for acute medical treatment, as follows. Mild: outpatient care; moderate: hospitalisation without respiratory support; severe: hospitalisation with the need for oxygen supply; critical: patients treated at the intensive care unit with the need for noninvasive or invasive ventilation. Except for patients with mild COVID-19, who demonstrated only minor pulmonary CT abnormalities, all other patient groups demonstrated a significant improvement of lung abnormalities in CT scans ( $p=0.042$ to $p<0.001$ for time-dependent changes). CT severity scoring ranges from 0 to 25 and was applied as detailed in the methods section. Visit 1 (V1) and visit 2 (V2) were performed 60 and 100 days after the diagnosis of COVID-19, respectively. Data are presented as mean \pm SE. 


\section{Conclusion}

In summary, we describe significant residual clinical, functional and CT-morphological changes in the majority of COVID-19 patients, which dramatically improved within the observation period of 3 months. We conclude that follow-up care should be considered for patients with persisting clinical symptoms after COVID-19, which may include serial measures of lung function, echocardiography and CT scans of the chest.

Acknowledgements: We acknowledge the dedication, commitment and sacrifice of the staff, providers and personnel in our institutions through the COVID-19 crisis and the suffering and loss of our patients as well as in their families and our community.

This study is registered at ClinicalTrials.gov with identifier number NCT04416100. Study protocol will be available immediately following publication for anyone who wishes to access. Individual data will not be made publicly available.

Author contributions: T. Sonnweber, J. Löffler-Ragg, S. Sahanic and I. Tancevski designed the study. T. Sonnweber, S. Sahanic, A. Pizzini, G. Weiss, A. Luger, C. Schwabl, K. Kurz, S. Koppelstätter, D. Haschka, V. Petzer, B. Sonnweber, A. Boehm, M. Aichner, D. Lener, M. Theurl, A. Lorsbach-Köhler, A. Tancevski, A. Schapfl, M. Schaber, R. Hilbe, M. Nairz, B. Puchner, D. Hüttenberger, C. Tschurtschenthaler, M. Aßhoff, A. Peer, F. Hartig, R. Bellmann, M. Joannidis, C. Gollmann-Tepeköylü, J. Holfeld, G. Feuchtner, A. Egger, G. Hoermann, A. Schroll, G. Fritsche, S. Wildner, R. Bellmann-Weiler, R. Kirchmair, R. Helbok, E. Wöll, G. Widmann, J. Löffler-Ragg and I. Tancevski performed the clinical investigations and collected the data. T. Sonnweber, G. Widmann, A. Luger, C. Schwabl, P. Tymoszuk, G. Feuchtner, H. Prosch, D. Rieder, Z. Trajanoski and F. Kronenberg performed data analysis. T. Sonnweber, S. Sahanic, A. Pizzini, G. Widmann, A. Luger, C. Schwabl, P. Tymoszuk, D. Rieder, Z. Trajanoski, F. Kronenberg, G. Weiss, J. Löffler-Ragg and I. Tancevski interpreted data. T. Sonnweber, G. Weiss, H. Prosch, F. Kronenberg, R. Kirchmair, G. Widmann, J. Löffler-Ragg and I. Tancevski wrote the manuscript. All authors critically reviewed the final version of the manuscript.

Conflict of interest: T. Sonnweber has nothing to disclose. S. Sahanic has nothing to disclose. A. Pizzini has nothing to disclose. A. Luger has nothing to disclose. C. Schwabl has nothing to disclose. B. Sonnweber has nothing to disclose. K. Kurz has nothing to disclose. S. Koppelstätter has nothing to disclose. D. Haschka has nothing to disclose. V. Petzer has nothing to disclose. A. Boehm has nothing to disclose. M. Aichner has nothing to disclose. P. Tymoszuk has nothing to disclose. D. Lener has nothing to disclose. M. Theurl has nothing to disclose. A. Lorsbach-Köhler has nothing to disclose. A. Tancevski has nothing to disclose. A. Schapfl has nothing to disclose. M. Schaber has nothing to disclose. R. Hilbe has nothing to disclose. M. Nairz has nothing to disclose. B. Puchner has nothing to disclose. D. Hüttenberger has nothing to disclose. C. Tschurtschenthaler has nothing to disclose. M. Aßhoff has nothing to disclose. A. Peer has nothing to disclose. F. Hartig has nothing to disclose. R. Bellmann has nothing to disclose. M. Joannidis has nothing to disclose. C. Gollmann-Tepeköylü has nothing to disclose. J. Holfeld has nothing to disclose. G. Feuchtner has nothing to disclose. A. Egger has nothing to disclose. G. Hoermann has nothing to disclose. A. Schroll has nothing to disclose. G. Fritsche has nothing to disclose. S. Wildner has nothing to disclose. R. Bellmann-Weiler has nothing to disclose. R. Kirchmair has nothing to disclose. R. Helbok has nothing to disclose. H. Prosch has nothing to disclose. D. Rieder has nothing to disclose. Z. Trajanoski has nothing to disclose. F. Kronenberg has nothing to disclose. E. Wöll has nothing to disclose. G. Weiss has nothing to disclose. G. Widmann has nothing to disclose. J. Löffler-Ragg has nothing to disclose. I. Tancevski reports an Investigator Initiated Study (IIS) grant from Boehringer Ingelheim (IIS 1199-0424).

Support statement: This study was supported by the Austrian National Bank Fund (Project 17271, J. Löffler-Ragg), and the "Verein zur Förderung von Forschung und Weiterbildung in Infektiologie und Immunologie, Innsbruck" (G. Widmann). Additionally, this study was supported by Boehringer Ingelheim RCV GmbH \& Co KG (BI). BI had no role in the design, analysis or interpretation of the results in this study. BI was given the opportunity to review the manuscript for medical and scientific accuracy as it relates to BIPI substances, as well as intellectual property considerations. Funding information for this article has been deposited with the Crossref Funder Registry.

\section{References}

1 Huang C, Wang Y, Li X, et al. Clinical features of patients infected with 2019 novel coronavirus in Wuhan, China. Lancet 2020; 395: 497-506.

2 Hui DS, Joynt GM, Wong KT, et al. Impact of severe acute respiratory syndrome (SARS) on pulmonary function, functional capacity and quality of life in a cohort of survivors. Thorax 2005; 60: 401-409.

3 Blanco-Melo D, Nilsson-Payant BE, Liu WC, et al. Imbalanced host response to SARS-CoV-2 drives development of COVID-19. Cell 2020; 181: 1036-1045.

4 Moore JB, June CH. Cytokine release syndrome in severe COVID-19. Science 2020; 368: 473-474.

5 McGonagle D, Sharif K, O’Regan A, et al. The role of cytokines including interleukin-6 in COVID-19 induced pneumonia and macrophage activation syndrome-like disease. Autoimmun Rev 2020; 19: 102537.

6 Peiris JS, Chu CM, Cheng VC, et al. Clinical progression and viral load in a community outbreak of coronavirus-associated SARS pneumonia: a prospective study. Lancet 2003; 361: 1767-1772.

7 Walls AC, Park YJ, Tortorici MA, et al. Structure, function, and antigenicity of the SARS-CoV-2 spike glycoprotein. Cell 2020; 181: 281-292.

8 Jaimes JA, André NM, Chappie JS, et al. Phylogenetic analysis and structural modeling of SARS-CoV-2 spike protein reveals an evolutionary distinct and proteolytically sensitive activation loop. J Mol Biol 2020; 432: 3309-3325.

9 Monteil V, Kwon H, Prado P, et al. Inhibition of SARS-CoV-2 infections in engineered human tissues using clinical-grade soluble human ACE2. Cell 2020; 181: 905-913. 
10 Imai Y, Kuba K, Penninger JM. The discovery of angiotensin-converting enzyme 2 and its role in acute lung injury in mice. Exp Physiol 2008; 93: 543-548.

11 Imai $\mathrm{Y}, \mathrm{Kuba} \mathrm{K}$, Rao S, et al. Angiotensin-converting enzyme 2 protects from severe acute lung failure. Nature 2005; 436: 112-116.

$12 \mathrm{Fu} \mathrm{Y}$, Cheng Y, Wu Y. Understanding SARS-CoV-2-mediated inflammatory responses: from mechanisms to potential therapeutic tools. Virol Sin 2020; 35: 266-271.

13 Kuba K, Imai Y, Rao S, et al. A crucial role of angiotensin converting enzyme 2 (ACE2) in SARS coronavirus-induced lung injury. Nat Med 2005; 11: 875-879.

14 Hui DS, Wong KT, Ko FW, et al. The 1-year impact of severe acute respiratory syndrome on pulmonary function, exercise capacity, and quality of life in a cohort of survivors. Chest 2005; 128: 2247-2261.

$15 \mathrm{Ng}$ CK, Chan JW, Kwan TL, et al. Six month radiological and physiological outcomes in severe acute respiratory syndrome (SARS) survivors. Thorax 2004; 59: 889-891.

16 Ngai JC, Ko FW, Ng SS, et al. The long-term impact of severe acute respiratory syndrome on pulmonary function, exercise capacity and health status. Respirology 2010; 15: 543-550.

17 Das KM, Lee EY, Singh R, et al. Follow-up chest radiographic findings in patients with MERS-CoV after recovery. Indian J Radiol Imaging 2017; 27: 342-349.

18 World Health Organization (WHO). Coronavirus. 2020. www.who.int/health-topics/coronavirus\#tab=tab_3 Date last accessed: October 20, 2020.

19 Hansell DM, Bankier AA, MacMahon H, et al. Fleischner Society: glossary of terms for thoracic imaging. Radiology 2008; 246: 697-722.

20 Rees EM, Nightingale ES, Jafari Y, et al. COVID-19 length of hospital stay: a systematic review and data synthesis. $B M C$ Med 2020; 18: 270 .

21 Mo X, Jian W, Su Z, et al. Abnormal pulmonary function in COVID-19 patients at time of hospital discharge. Eur Respir J 2020; 55: 2001217.

22 Frija-Masson J, Debray MP, Gilbert M, et al. Functional characteristics of patients with SARS-CoV-2 pneumonia at 30 days post-infection. Eur Respir J 2020; 56: 2001754.

23 Richardson S, Hirsch JS, Narasimhan M, et al. Presenting characteristics, comorbidities, and outcomes among 5700 patients hospitalized with COVID-19 in the New York City Area. JAMA 2020; 323: 2052-2059.

24 Grasselli G, Zangrillo A, Zanella A, et al. Baseline characteristics and outcomes of 1591 patients infected with SARS-CoV-2 admitted to ICUs of the Lombardy region, Italy. JAMA 2020; 323: 1574-1581.

25 Zhou F, Yu T, Du R, et al. Clinical course and risk factors for mortality of adult inpatients with COVID-19 in Wuhan, China: a retrospective cohort study. Lancet 2020; 395: 1054-1062.

26 Klein SJ, Fries D, Kaser S, et al. Unrecognized diabetes in critically ill COVID-19 patients. Crit Care 2020; $24: 406$.

27 Zhang $\mathrm{P}, \mathrm{Li} \mathrm{J}$, Liu $\mathrm{H}$, et al. Long-term bone and lung consequences associated with hospital-acquired severe acute respiratory syndrome: a 15-year follow-up from a prospective cohort study. Bone Res 2020; 8: 8 .

28 Herridge MS, Cheung AM, Tansey CM, et al. One-year outcomes in survivors of the acute respiratory distress syndrome. N Engl J Med 2003; 348: 683-693.

29 Cheung AM, Tansey CM, Tomlinson G, et al. Two-year outcomes, health care use, and costs of survivors of acute respiratory distress syndrome. Am J Respir Crit Care Med 2006; 174: 538-544.

30 Wang Y, Dong C, Hu Y, et al. Temporal changes of CT findings in 90 patients with COVID-19 pneumonia: a longitudinal study. Radiology 2020; 296: E55-E64.

31 Suliman YA, Dobrota R, Huscher D, et al. Brief report: pulmonary function tests: high rate of false-negative results in the early detection and screening of scleroderma-related interstitial lung disease. Arthritis Rheumatol 2015; 67: 3256-3261.

32 Burnham EL, Janssen WJ, Riches DW, et al. The fibroproliferative response in acute respiratory distress syndrome: mechanisms and clinical significance. Eur Respir J 2014; 43: 276-285. 